\title{
III-V diluted magnetic semiconductor: Substitutional doping of Mn in InAs
}

\author{
Y. L. Soo, S. W. Huang, Z. H. Ming, and Y. H. Kao \\ Department of Physics, State University of New York at Buffalo, Amherst, New York 14260 \\ H. Munekata \\ Imaging Science and Engineering Laboratory, Tokyo Institute of Technology, Yokohama 226, Japan \\ L. L. Chang \\ Hong Kong University of Science and Technology, Kowloon, Hong Kong \\ (Received 26 April 1995; revised manuscript received 19 October 1995)
}

\begin{abstract}
Local structures around $\mathrm{Mn}$ in $\mathrm{In}_{1-x} \mathrm{Mn}_{x}$ As films grown by molecular-beam epitaxy have been studied by using Mn $K$-edge extended x-ray-absorption fine-structure (EXAFS) technique. Substitution of Mn atoms for the In sites is found in samples either grown at low substrate temperatures (near $200{ }^{\circ} \mathrm{C}$ ) or with a low $\mathrm{Mn}$ concentration (about 1 at. \%). This result represents a significant extension of an earlier EXAFS study and serves as direct experimental evidence for III-V diluted magnetic semiconductors obtained by substitutional doping of Mn impurities in InAs.
\end{abstract}

\section{INTRODUCTION}

Incorporation of a controllable large amount of magnetic impurities into the III-V semiconductor InAs was first accomplished in 1989 by using molecular-beam-epitaxy (MBE) techniques. ${ }^{1}$ This result demonstrated a fact that it is now possible to synthesize III-V magnetic semiconductors under proper MBE processing conditions. These materials could become potentially useful for technical applications. Subsequent measurements of these $\operatorname{In}_{1-x} \mathrm{Mn}_{x}$ As films prepared with various $\mathrm{Mn}$ contents and at different substrate temperatures have shown three characteristic types of transport and magnetic properties, ${ }^{2-4}$ these are summarized schematically in Fig. 1. It is conceivable that these different physical properties are closely related to the local environment surrounding the impurity atoms, and the local disorder could have an important effect on the magnetic coupling between Mn ions. For an understanding of the basic properties of this new class of magnetic semiconductors regarding the type of carriers, degree of compensation, ${ }^{5}$ and microstructures (e.g., formation of complexes or phase separation) as a result of $\mathrm{Mn}$ doping, it is desirable to obtain a clear physical picture of the local structures around the Mn atoms.

We have previously investigated the local structures around $\mathrm{Mn}$ in $\mathrm{In}_{1-x} \mathrm{Mn}_{x}$ As films with a specific value of $x=0.12$ grown at high and low substrate temperature $T_{s}$ using the extended $\mathrm{x}$-ray-absorption fine-structure (EXAFS) technique. ${ }^{6}$ However, the $\mathrm{Mn}-\mathrm{As}_{6}-$ complex model considered earlier for the low- $T_{s}$ samples suffers from an ambiguity due to insufficient data. This ambiguity arises from the noncentral $\mathrm{Mn}$ atoms in a $\mathrm{Mn}-\mathrm{As}_{6}$ complex that contains six As nearest neighbors at $2.58 \AA$ and two Mn next-nearest neighbors at $2.83 \AA$ from the central $\mathrm{Mn}$ atom. In principle, these noncentral $\mathrm{Mn}$ atoms could have a local structure different from that of the central $\mathrm{Mn}$ atom. Reconsideration of the previous model with improved data and analysis is necessary.

In the present work, we have pursued a more thorough study of the local environment surrounding the Mn atoms using a variety of growth parameters in the $\operatorname{In}_{1-x} \mathrm{Mn}_{x}$ As films. Four additional samples of $\operatorname{In}_{1-x} \mathrm{Mn}_{x}$ As grown at different substrate temperatures and with Mn concentrations $x$ pertaining to the above-mentioned three different types of behavior (see Fig. 1) were investigated in detail using EXAFS. The data were analyzed with an improved curvefitting method. For comparison, we have measured EXAFS spectra of a polycrystalline MnAs film that was also used as a model compound in the data analysis. The structure of this model compound was well characterized by x-ray diffraction and found in good agreement with that of bulk MnAs. In addition, a low- $T_{s}$ film annealed at $480{ }^{\circ} \mathrm{C}$ was also examined to investigate the effects of annealing on these films.

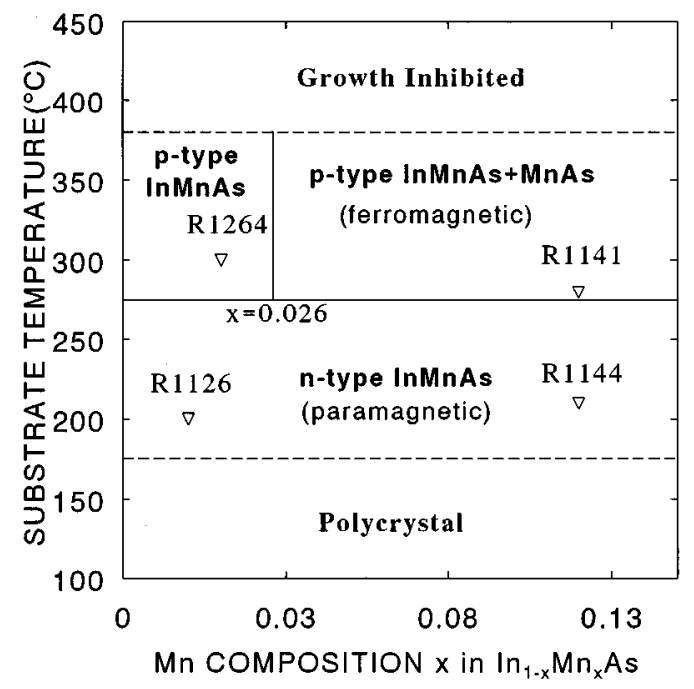

FIG. 1. Schematic diagram of the film properties and their relation to the growth parameters (substrate temperature and Mn concentration). Points representing (In,Mn)As films used in the present experiment are indicated with $\nabla$ marks along with their corresponding sample numbers (see also Table I). 
TABLE I. A list of samples used in the present EXAFS experiment.

\begin{tabular}{|c|c|c|c|c|}
\hline Sample & Chemical formula & Growth temperature $\left({ }^{\circ} \mathrm{C}\right)$ & Carrier type & Magnetism \\
\hline$R 1201$ & MnAs & & & ferromagnetic \\
\hline $\begin{array}{l}R 1144 A \\
\text { (annealed) }\end{array}$ & $\mathrm{In}_{0.88} \mathrm{Mn}_{0.12} \mathrm{As}$ & $\begin{array}{c}210 \\
\left(\text { annealed at } 480^{\circ} \mathrm{C}\right)\end{array}$ & & \\
\hline$R 1141$ & $\mathrm{In}_{0.88} \mathrm{Mn}_{0.12} \mathrm{As}$ & 280 & & ferromagnetic \\
\hline$R 1144$ & $\mathrm{In}_{0.88} \mathrm{Mn}_{0.12} \mathrm{As}$ & 210 & $n$ type & paramagnetic \\
\hline$R 1126$ & $\mathrm{In}_{0.986} \mathrm{Mn}_{0.014} \mathrm{As}$ & 200 & $n$ type & paramagnetic \\
\hline$R 1264$ & $\mathrm{In}_{0.983} \mathrm{Mn}_{0.017} \mathrm{As}$ & 300 & $p$ type & paramagnetic \\
\hline
\end{tabular}

\section{EXPERIMENTAL AND DATA ANALYSIS}

Samples of $\mathrm{In}_{1-x} \mathrm{Mn}_{x}$ As films were grown by MBE at both low $\left(200-210^{\circ} \mathrm{C}\right)$ and high $\left(280-300{ }^{\circ} \mathrm{C}\right)$ substrate temperatures. ${ }^{7}$ One of the low- $T_{s}$ films was then annealed at $480{ }^{\circ} \mathrm{C}$ for $74 \mathrm{~min}$ in the $\mathrm{MBE}$ chamber under $\mathrm{As}_{4}$ beam flux. A model compound polycrystalline MnAs film was also grown at $T_{s}=200{ }^{\circ} \mathrm{C}$. The composition and other characteristics of the six samples studied in the present experiment are summarized in Table I. The EXAFS measurements were performed at beamline X3B1 at the National Synchrotron Light Source using a conventional setup and standard procedures. ${ }^{8}$ Except for the annealed sample $\operatorname{In}_{0.88} \mathrm{Mn}_{0.12} \mathrm{As}$ (R1144A), which was measured at room temperature, all the other films were measured at $40 \mathrm{~K}$ in order to reduce the Debye-Waller factor.

Established background-subtraction and correction methods were used to extract the EXAFS $\chi$ functions from the raw data. ${ }^{8,9}$ The $\chi$ functions were then weighted with $k$ and Fourier transformed into the real space for detailed comparison $^{10}$ (shown by fine lines in Fig. 2). In view of the distinct patterns of the oscillations, especially in the region

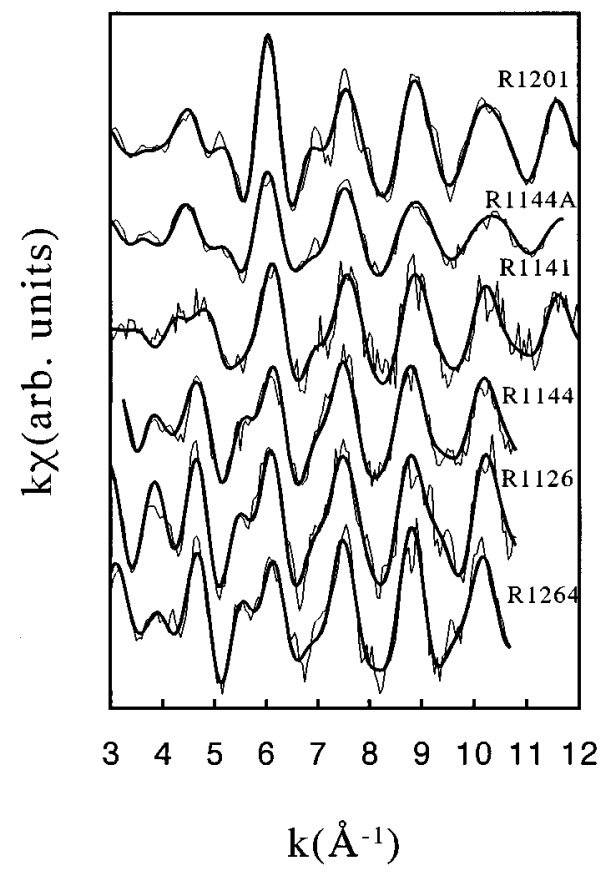

FIG. 2. Comparison of experimental $k \chi$ functions (fine lines) with calculated EXAFS results (coarse lines) for all six samples using the final values of curve-fitting parameters listed in Table III. between 3.0 and $6.0 \AA^{-1}$, the results can clearly be classified into two separate groups, representing two distinct types of local structures around the central $\mathrm{Mn}$ atom. The first group includes the model compound MnAs (R1201), an annealed low- $T_{s}$ high-concentration sample $\operatorname{In}_{0.88} \mathrm{Mn}_{0.12} \mathrm{As}(R 1144 A)$, and a high- $T_{s}$ high-concentration $\operatorname{In}_{0.88} \mathrm{Mn}_{0.12} \mathrm{As}$ (R1141). The second group includes an as-grown low- $T_{s}$ highconcentration sample $\operatorname{In}_{0.88} \mathrm{Mn}_{0.12} \mathrm{As}(R 1144)$, a low- $T_{s}$ lowconcentration $\operatorname{In}_{0.986} \mathrm{Mn}_{0.014} \mathrm{As}(R 1126)$, and a high- $T_{s}$ lowconcentration $\mathrm{In}_{0.983} \mathrm{Mn}_{0.017} \mathrm{As}$ (R1264). The corresponding Fourier transforms of these EXAFS patterns are shown in Fig. 3, which also exhibit characteristic differences between the two groups consistent with the $k$-space classification. Figure 3 also reveals some variations within the same group: (i) A peak at $\sim 4.0 \AA$ in the first group data for $R 1201$ and $R 1144 A$ is absent in $R 1141$. (ii) A shoulder at $\sim 2.8 \AA$ on the high- $R$ side of the first peak for $R 1264$ is absent in the other two samples in the second group.

The experimental data were analyzed and compared with theoretical calculations using an improved curve-fitting

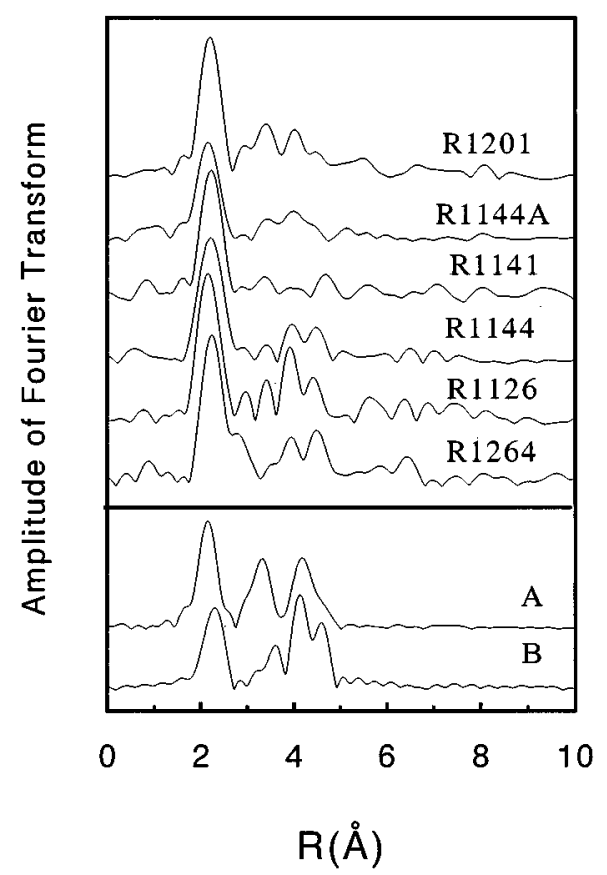

FIG. 3. Comparison of the magnitude of Fourier transform obtained in the present experiment (upper panel) with model calculations based on the MnAs structure (A) and InAs structure (B) using parameters given in Table II (lower panel). 
TABLE II. Structural parameters for MnAs and InAs ( $N$ is the coordination number, and $R$ is the interatomic distance).

\begin{tabular}{lcrc}
\hline \hline Central atom & Neighboring atom & $N$ & $R(\AA)$ \\
\hline Mn in MnAs & As & 6 & 2.57 \\
& Mn & 2 & 2.85 \\
Mn & 6 & 3.71 \\
& As & 6 & 4.51 \\
Mn & 12 & 4.68 \\
In in InAs & As & 6 & 4.78 \\
& As & 4 & 2.61 \\
& In & 12 & 4.27 \\
& As & 12 & 5.00 \\
\hline \hline
\end{tabular}

method. ${ }^{8,9,11}$ Three major steps were taken in this curvefitting procedure. First, using the known structures of MnAs and InAs (see Table II), we calculated theoretical Mn $K$-edge EXAFS spectra to initiate the curve-fitting process based on two most probable models: (A) an MnAs local structure, or (B) an In-site-substitution structure. ${ }^{12}$ A direct comparison of the Fourier transform of these calculated spectra (see lower panel in Fig. 3) and that of the sample under consideration can give an unequivocal choice of either (A) or (B), but not both. In the event that the final curve-fitting result is unsatisfactory, the initially selected model was then modified by either allowing the structural parameters to vary, or by including additional physically conceivable near-neighbor shells, and/or by introducing a non-Gaussian local disorder in terms of the cumulants $\mathrm{C}_{3}$ and $\mathrm{C}_{4} \cdot{ }^{13}$ A satisfactory fit was then established for each sample studied. In the second step, as a double check, we challenged the uniqueness of the model adopted in the first step (say, A or B) by a forced curve fitting of the same data with the other most probable model (B or A). Such a forced curve fitting always gives unphysical or inconsistent results for all of our samples, implying that the structural model established in the first step is indeed superior to the other alternative. In the third step, we further tested whether the structural model excluded in the second step could possibly exist as a minority phase in the sample by performing a two-phase curve fitting that determines the allowed fraction of the minority phase in each sample. In this procedure, we have used parameters obtained in the first-step curve fitting for samples $R 1201$ and $R 1126$ to calculate the $\chi$ function for the possible minority-phase MnAs and In-sitesubstitution structures, respectively. It turns out that only the low- $T_{s}$ high-concentration sample $(R 1144)$ and the annealed sample $(R 1144 A)$ could allow a nontrivial content of the minority phase.

\section{RESULTS AND DISCUSSION}

Through detailed sample-by-sample EXAFS analyses, an unequivocal choice between the two most probable local structural models (A) or (B) can be clearly made for each sample studied (with some minor modifications in the special cases of $R 1144$ and $R 1144 A$ ). The final values of structural parameters obtained from these detailed analyses are summarized in Table III. Further, in order to identify the peaks in the Fourier transform magnitude with various neighboring shells around $\mathrm{Mn}$, an "anatomy" of the curve-fitting results

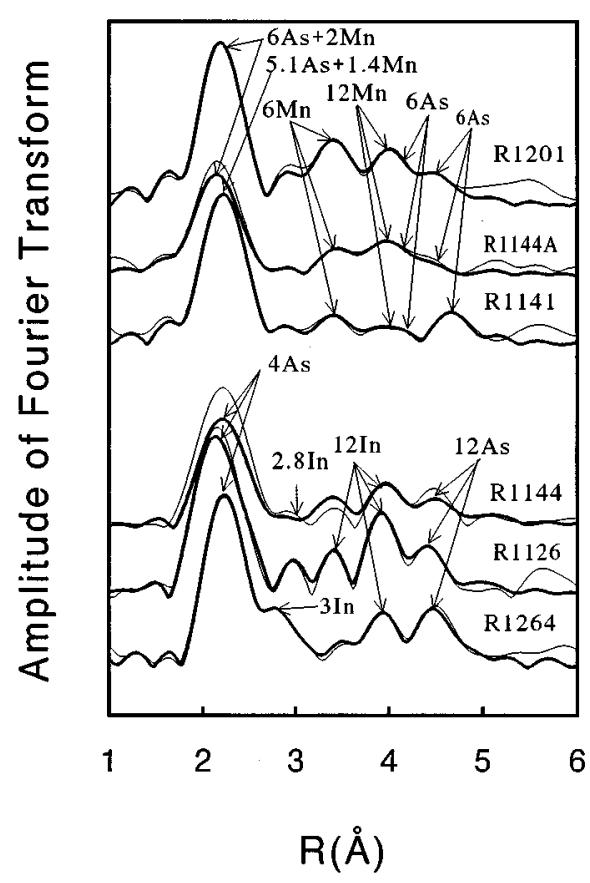

FIG. 4. A summary of the anatomy results for all six samples (coarse lines) in comparison with experimental results (fine lines). Note that for samples containing a significant amount of minority phases (MnAs in $R 1144$ and In-site substitution in $R 1144 A$ ), only the majority-phase components in the calculated magnitude of Fourier transform (coarse lines) are shown in the figure. Differences between data and calculation in these two samples also serve as an indication of the significant contributions from the minority phase.

was made by calculating the contribution from each individual shell using the parameters given in Table III. This shell anatomy is shown in Fig. 4.

By a comparison of Tables II and III (and also Fig. 4), it is evident that three samples in the first group $(R 1201-$ $R 1141$ ) all show a local structure around Mn similar to that in MnAs (NiAs-type structure) while the other three samples in the second group $(R 1144-R 1264)$ all show a majority local structure around $\mathrm{Mn}$ in good agreement with the Insubstitution model in which the Mn atoms occupy the In sites in the zinc-blende structure of an InAs host. However, owing to the changes in Mn concentration and substrate temperature, the local structures around $\mathrm{Mn}$ for samples in the same group still show some minor variations. In the first group, in which the MnAs-like structure prevails, the annealed sample $(R 1144 A)$ shows large non-Gaussian local disorder. Further, $7.5 \%$ of $\mathrm{Mn}$ atoms in this sample could substitute for the In sites in the InAs host and form a minority phase. Also, the structure of the high- $T_{s}$ high-concentration sample (R1141), compared to that of MnAs, shows a significant increase in the interatomic distance and a decreased coordination number for the second (Mn) shell, as well as a decrease in both the interatomic distance and coordination number of the first (As) shell. In the second group, the nearest-neighbor distance between Mn and As (2.54-2.58 $\mathrm{A})$ is shorter than the In-As bond length $(2.61 \AA)$ in the InAs host (see Table II). Also, an additional In shell exists at a distance $3.11 \AA$ from the Mn impurity with a coordination number of about 3 in the high- $T_{s}$ low-concentration sample 
TABLE III. Local structures around Mn for each sample obtained from curve fitting. Underlined values were kept constant during fitting. Uncertainties of the last digit were estimated by the double-minimum residue $\left(2 \chi^{2}\right)$ method and shown in parentheses.

\begin{tabular}{|c|c|c|c|c|c|c|c|c|c|c|}
\hline Sample & $\begin{array}{c}R_{\min }-R_{\max } \\
(\AA)\end{array}$ & Atom & $N$ & $\begin{array}{c}R \\
(\AA)\end{array}$ & $\begin{array}{c}\sigma^{2}\left(C_{2}\right) \\
\left(10^{-3} \AA^{2}\right)\end{array}$ & $\begin{array}{c}C_{3} \\
\left(10^{-4} \AA^{3}\right)\end{array}$ & $\begin{array}{c}C_{4} \\
\left(10^{-5} \AA^{4}\right)\end{array}$ & $\begin{array}{l}\Delta E_{0} \\
(\mathrm{eV})\end{array}$ & $\begin{array}{c}\text { Weighting } \\
(\%)\end{array}$ & $\begin{array}{c}\chi^{2} \\
\left(10^{-3}\right)\end{array}$ \\
\hline \multirow[t]{6}{*}{$R 1201$} & \multirow[t]{6}{*}{$1.42-4.76$} & As & 6 & 2.57 & $3(3)$ & & & $2.0(9)$ & 100 & 5.68 \\
\hline & & $\mathrm{Mn}$ & $\underline{2}$ & 2.85 & $5(3)$ & & & $2(4)$ & & \\
\hline & & Mn & $\overline{6}$ & $\overline{3.71}$ & $6(3)$ & & & $0(3)$ & & \\
\hline & & As & $\underline{6}$ & $\overline{4.51}$ & $3(6)$ & & & $0(5)$ & & \\
\hline & & $\mathrm{Mn}$ & $\overline{12}$ & $\overline{4.68}$ & $9(6)$ & & & $-5(5)$ & & \\
\hline & & As & 6 & $\overline{4.78}$ & $7(40)$ & & & $8(6)$ & & \\
\hline \multirow[t]{9}{*}{$R 1144 A$} & \multirow[t]{9}{*}{$1.36-4.76$} & As & 6.1 & $2 . \overline{57(1)}$ & $7.6(8)$ & $5(3)$ & $3(26)$ & -1.7 & 92.5 & 0.13 \\
\hline & & Mn & $1.4(4)$ & 2.88 & $19(5)$ & $-33(5)$ & 31 & $5(4)$ & & \\
\hline & & $\mathrm{Mn}$ & $\underline{6}$ & 3.71 & $19(1)$ & & & -0.6 & & \\
\hline & & As & $\underline{6}$ & $\overline{4.51}$ & $9(2)$ & & & $-2(3)$ & & \\
\hline & & $\mathrm{Mn}$ & $\underline{12}$ & $\overline{4.68}$ & $13(2)$ & & & $-7.1 \quad(5)$ & & \\
\hline & & As & $\underline{6}$ & 4.78 & 40 & & & $9(15)$ & & \\
\hline & & As & $\underline{4}$ & 2.54 & $\underline{0.1}$ & & & -7 & 7.5 & \\
\hline & & In & $\underline{12}$ & 4.22 & $\underline{8}$ & & & -11 & & \\
\hline & & As & $\overline{12}$ & $\overline{5.01}$ & $\overline{6}$ & & & $\overline{-7}$ & & \\
\hline \multirow[t]{6}{*}{$R 1141$} & \multirow[t]{6}{*}{$1.76-5.16$} & As & $5(1)$ & $2.56(2)$ & $3(3)$ & & & $1(4)$ & 100 & 3.99 \\
\hline & & $\mathrm{Mn}$ & $1(4)$ & $2.98(5)$ & $9(35)$ & & & $13(10)$ & & \\
\hline & & $\mathrm{Mn}$ & $\underline{6}$ & $\underline{3.71}$ & $15(5)$ & & & $1(4)$ & & \\
\hline & & As & $\underline{6}$ & 4.51 & $6(4)$ & & & $3(4)$ & & \\
\hline & & $\mathrm{Mn}$ & $\overline{12}$ & $\overline{4.68}$ & $37(40)$ & & & $-5(5)$ & & \\
\hline & & As & $\underline{6}$ & 4.78 & $3(4)$ & & & $12(2)$ & & \\
\hline \multirow[t]{10}{*}{$R 1144$} & \multirow[t]{10}{*}{$1.64-4.90$} & As & $\overline{4}$ & $2 . \overline{57(1)}$ & 0.4 (4) & & & -1 & 74.5 & 0.83 \\
\hline & & In & 2.8 & 3.00 & $6(3)$ & & & $24(1)$ & & \\
\hline & & In & 12 & $4.25(3)$ & $11(2)$ & & & $-5.7(5)$ & & \\
\hline & & As & $\overline{12}$ & 5.06 & $6(2)$ & & & $1(5)$ & & \\
\hline & & As & $\underline{6}$ & $\underline{2.57}$ & $\underline{3}$ & & & $\underline{2}$ & 25.5 & \\
\hline & & Mn & $\underline{2}$ & $\overline{2.85}$ & $\overline{5}$ & & & $\overline{2}$ & & \\
\hline & & $\mathrm{Mn}$ & $\underline{6}$ & 3.71 & $\underline{6}$ & & & $\underline{0}$ & & \\
\hline & & As & $\overline{6}$ & $\overline{4.51}$ & $\overline{3}$ & & & $\underline{0}$ & & \\
\hline & & $\mathrm{Mn}$ & $\underline{12}$ & $\overline{4.68}$ & $\underline{9}$ & & & -5 & & \\
\hline & & As & 6 & 4.78 & $\overline{7}$ & & & $\underline{8}$ & & \\
\hline \multirow[t]{3}{*}{$R 1126$} & \multirow[t]{3}{*}{$1.64-4.90$} & As & $\underline{4}$ & $2 . \overline{54(3)}$ & $0.1^{-}(8)$ & & & $-7^{-}(5)$ & 100 & 23.3 \\
\hline & & In & $\underline{12}$ & $4.22(4)$ & $8(5)$ & & & -11 & & \\
\hline & & As & $\overline{12}$ & 5.01 & $6(7)$ & & & $-7(43)$ & & \\
\hline \multirow[t]{4}{*}{$R 1264$} & \multirow[t]{4}{*}{$1.74-5.0$} & As & $\underline{4}$ & $2.58(2)$ & $0.2(5)$ & & & $0(4)$ & 100 & 2.88 \\
\hline & & In & $3(1)$ & 3.11 & $4(5)$ & & & $-9(5)$ & & \\
\hline & & In & $\underline{12}$ & 4.16 & $13(3)$ & & & $-11(5)$ & & \\
\hline & & As & $\underline{12}$ & 5.00 & $4(3)$ & & & $-7(5)$ & & \\
\hline
\end{tabular}

(R1264). The appearance of this additional In shell is accompanied by a shortened Mn-In bond length (4.16 $\AA$ ) compared to the In-In distance $(4.27 \AA)$ in the original InAs structure. In addition to this obvious structural variation in $R 1264$, which appears as a shoulder in the Fourier transform, we have found it necessary to include an additional In neighboring shell with a coordination number of 2.8 at a distance of $3.00 \AA$ in $R 1144$ in order to obtain a reasonable fit for the high- $R$ region. A significant $25.5 \%$ of $\mathrm{Mn}$ atoms in sample $R 1144$ may be in the form of MnAs clusters according to the results of two-phase fitting. With our improved EXAFS analysis method, the results of this low- $T_{s}$ highconcentration sample $R 1144$ are largely different from the $\mathrm{Mn}^{-\mathrm{As}_{6}}$-complex model proposed earlier. $^{6}$

It is remarkable that the low-concentration sample $R 1264$ prepared at high- $T_{s}$ contains an extra shell (see the "shoulder" in Fig. 3) with three In atoms at $3.11 \AA$ in addition to the usual near neighbors in InAs. The presence of this extra In shell in the EXAFS data could arise from either a variation of the local structure around $\mathrm{Mn}$ atoms that occupy the In sites or a possible coexistence of other Mn atoms with different local structures. In contrast to other samples in the second group that are $n$ type, this particular film is $p$ type. One might speculate that the difference of carrier type in this sample may owe its origin to the changes in the local structure around the Mn atoms.

\section{CONCLUSION}

The local structures around Mn impurities derived from our EXAFS data provide useful information on the physical 
consequences of Mn doping under various processing conditions and with different Mn concentrations. Our results provide clean-cut evidence that substitution of Mn for the In site in the InAs host can indeed be achieved by using a low Mn concentration (with $x$ around 0.01) and at low substrate temperatures $\left(T_{s} \sim 200{ }^{\circ} \mathrm{C}\right)$. Either increasing $x$ to $\sim 0.1$ or raising $T_{s}$ to $300{ }^{\circ} \mathrm{C}$ may still result in predominantly In-site substitution, but accompanied by an inevitable local structural modification (e.g., the appearance of an additional In shell of $\sim 3$ In atoms at $\sim 3 \AA$ from the $\mathrm{Mn}$ atom). These results demonstrate that III-V diluted magnetic semiconductors can be prepared by substitutional doping of magnetic impurities under proper MBE processing conditions. Since the local structure around the impurity atom can have a profound influence on its effective valency in the host, it could be difficult to control the $n$ or $p$ type of carriers by $\mathrm{Mn}$ doping if the low-concentration and low- $T_{s}$ conditions are not simultaneously fulfilled. On the other hand, in contrast to the In-site-substitution films, the high Mn-content films with $x>0.1$ either grown at high- $T_{s}$ (near $300{ }^{\circ} \mathrm{C}$ ) or at low- $T_{s}$ $\left(\sim 200^{\circ} \mathrm{C}\right)$ but annealed at an elevated temperature all contain a large amount of MnAs clusters; the resulting films are ferromagnetic and similar to polycrystalline MnAs. The technique of impurity control may provide unprecedented opportunities for making new III-V magnetic semiconductors.

\section{ACKNOWLEDGMENTS}

The present research at SUNY-Buffalo was supported by the U.S. Department of Energy under Grant No. DE-FG0287ER45283. The SUNY X3 beamline at NSLS is supported by the Division of Basic Energy Sciences of the U.S. Department of Energy (DE-FG02-86ER45231). The work at IBM was supported in part by the Army Research Office. The work at Tokyo Institute of Technology is supported in part by the TEPCO Research Foundation, the TORAY Science Foundation, the Grant-in-Aid for General Science Research No. 07455006, the Grant-in-Aid for Developmental Science Research No. 07555004, and the NEDO Research Project for the Development of New Materials.
${ }^{1}$ H. Munekata, H. Ohno, S. von Molnar, Armin Segmüller, L. L. Chang, and L. Esaki, Phys. Rev. Lett. 63, 1849 (1989).

${ }^{2}$ H. Munekata, H. Ohno, S. von Molnar, Alex Harwit, Armin Segmüller, and L. L. Chang, J. Vac. Sci. Technol. B 8, 176 (1990).

${ }^{3}$ H. Ohno, H. Munekata, S. von Molnar, and L. L. Chang, J. Appl. Phys. 69, 6103 (1991).

${ }^{4}$ S. von Molnar, H. Munekata, H. Ohno, and L. L. Chang, J. Magn. Magn. Mater. 93, 356 (1991).

${ }^{5}$ B. I. Shklovskii and A. L. Efros, Electronic Properties of Doped Semiconductors (Springer-Verlag, Berlin, 1984); C. Hilsum and A. C. Rose-Innes, Semiconducting III-V Compounds (Pergamon, New York, 1961).

${ }^{6}$ A. Krol, Y. L. Soo, S. Huang, Z. H. Ming, Y. H. Kao, H. Munekata, and L. L. Chang, Phys. Rev. B 47, 7187 (1993).

${ }^{7}$ S. Guha and H. Munekata, J. Appl. Phys. 74, 2974 (1993).
${ }^{8}$ Y. L. Soo, Z. H. Ming, S. W. Huang, Y. H. Kao, R. N. Bhargava, and D. Gallagher, Phys. Rev. B 50, 7602 (1994).

${ }^{9}$ M. Newville, P. Livin, Y. Yacoby, J. J. Rehr, and E. A. Stern, Phys. Rev. B 47, 14126 (1993).

${ }^{10}$ P. A. Lee, P. H. Citrin, P. Eisenberger, and B. M. Kincaid, Rev. Mod. Phys. 53, 760 (1981).

${ }^{11}$ J. J. Rehr, J. Mustre de Leon, S. I. Zabinsky, and R. C. Albers, J. Am. Chem. Soc. 113, 5135 (1991).

${ }^{12}$ In the calculations of $\chi$ functions to demonstrate the characteristic differences between the MnAs model and the In-site-substitution model, we have used the values of $N$ and $R$ given in Table II and imposed a common value of $\sigma^{2}=0.005 \AA^{2}$ to each neighboring shell up to $\sim 5 \AA$. See plots in the lower panel of Fig. 3 .

${ }^{13}$ G. Bunker, Nucl. Instrum. Methods Phys. Res. 207, 437 (1983). 